\title{
Hallazgos histopatológicos compatibles con enfermedad degenerativa valvular en perros asintomáticos
}

\author{
Gómez, L.O.'; Collazos, M.² \\ ${ }^{1}$ Grupo de investigación en cardiología, ${ }^{2}$ Cátedra de Patología UDCA, Facultad de Medicina Veterinaria y \\ Zootecnia, Universidad Antonio Nariño (UAN), Cra 3ra este \#47 ${ }^{\mathrm{a}}-15$, Bogotá, Colombia. \\ Tel: 00571-3384960 ext. 133. E.mail: leongomez@uan.edu.co.
}

\begin{abstract}
Resumen
Gómez, L.O.; Collazos, M.: Hallazgos histopatológicos compatibles con enfermedad degenerativa valvular en perros asintomáticos. Rev. vet. 21: 1, 13-18, 2010. El objetivo de esta investigación fue determinar los cambios histopatológicos de las aletas valvulares compatibles con enfermedad degenerativa valvular (EDV) en perros que al examen clínico se encontraban asintomáticos. La muestra fue de 38 corazones obtenidos en el Centro de Adopción y Tenencia de Mascotas de Bogotá sometidos al procedimiento de eutanasia como parte del control poblacional en el programa distrital contra la rabia. El 57,9\% de los animales correspondieron a machos y el $42,1 \%$ a hembras, principalmente mestizos $(n=21)$, Poodle $(n=15)$, Cocker Spaniel $(\mathrm{n}=1)$ y Schnauzer $(\mathrm{n}=1)$. El peso promedio fue de 7,34 $\pm 2,44 \mathrm{~kg}(3-13 \mathrm{~kg})$ y la edad promedio aproximada por evaluación dentaria fue $7 \pm 3$ años (2-15 años). Clínicamente, los animales no presentaron signos de compromiso cardíaco. La interpretación de los cambios histopatológicos se basó en la clasificación Kogure. Con la coloración de hematoxilina y eosina se encontró que un $65,8 \%$ de las válvulas septales mitrales y el $68,4 \%$ de las laterales presentaron cambios compatibles con EDV al igual que el 55,3\% de las septales tricuspídeas y el $84,2 \%$ de las laterales. Con la coloración alcian blue, especial para evaluar tejido conectivo, se encontró que el $76,3 \%$ de las aletas septales mitrales y el $76,3 \%$ de las laterales presentaron cambios compatibles con EDV. De igual manera al evaluar el aparato tricuspídeo se encontró que el $68,4 \%$ de sus aletas septales y el $65,8 \%$ de las laterales presentaron estos cambios. Esto demuestra que las lesiones del tejido valvular empiezan antes de la aparición del signo más frecuente de esta enfermedad que es el soplo. Por lo tanto se evidencia la necesidad de empezar a diseñar programas de medicina preventiva para reducir el riesgo de morbilidad y mortalidad de esta enfermedad debida a la remodelación ventricular como consecuencia de la activación neurohormonal temprana.
\end{abstract}

Palabras clave: perro, cardiología, degeneración valvular, histopatología, infiltración de mucopolisacáridos

\begin{abstract}
Gómez, L.O.; Collazos, M.: Histopathological changes compatible with degenerative valvular disease in asymptomatic dogs. Rev. vet. 21: 1, 13-18, 2010. The aim of this study was to determine the histopathological changes of leaflets compatible with canine chronic valvular heart disease (VHD) in asymptomatic dogs. The samples were obtained from 38 hearts of canines from the Holding and Adoption Pet Center of Bogotá, Colombia, submitted to euthanasia as part of the district canine population control to prevent rabies. Animals were $57.9 \%$ males, $42.1 \%$ females, mainly mixed breed $(n=21)$, Poodle $(n=15)$, Cocker Spaniel $(n$ $=1)$ and Schnauzer $(\mathrm{n}=1)$. Weight was $7.34 \pm 2.44 \mathrm{~kg}(3-13 \mathrm{~kg})$ and the average age determined by dental evaluation was $7 \pm 3$ years $(2-15$ years). The histopathology interpretation was based on the Kogure classification. It was found using hematoxylin-eosin stain that $65.8 \%$ of the septal mitral leaflets and $68.4 \%$ of the lateral leaflets presented changes compatible with VHD, as well as the $55.3 \%$ of the septal leaflets of tricuspid valve and $84.2 \%$ of the lateral leaflets. With the alcian blue coloration, indicated to evaluate connective tissue, it was found that $76.3 \%$ of the septal leaflets of mitral valve and $76.3 \%$ of the lateral leaflets presented changes compatible with VHD. In the same way, when the tricuspid valve was evaluated, it was found that $68.4 \%$ of septal leaflets and $65.8 \%$ of lateral leaflets presented these changes. This demonstrates that lesions of the valvular apparatus begin before the appearance of murmur, the most frequent sign for this disease. The need of preventive medicine programs to
\end{abstract}

Recibido: 2 junio 2010 / Aceptado: 28 junio 2010

Trabajo avalado por el Comité de Ética para Investigación Animal de la UAN en términos de la Ley 84/1989 sobre Bienestar Animal. 
reduce the morbility risk and mortality of this disease, related to ventricular remodeling as consequence of early neurohormonal activation, is emphasized.

Key words: dog, cardiology, valvular degeneration, histopathology, mucopolysaccharides infiltration.

\section{INTRODUCCIÓN}

La enfermedad degenerativa valvular (EDV), endocardiosis o enfermedad valvular crónica, es la patología cardiovascular con mayor prevalencia, $75 \%$ de los pacientes caninos llevados a consulta cardiológica son diagnosticados en diferentes estadios de la enferme$\mathrm{dad}^{2,9,11,14}$. La EDV se presenta con mayor frecuencia en animales adultos ( $>7$ años), de razas pequeñas como Poodle estándar y miniatura, Schnauzer, Pinscher, entre otras y mestizos menores de $15 \mathrm{~kg}$. En algunos casos se puede encontrar en razas como labrador o criollos de talla grande ${ }^{20}$.

Los cambios en el aparato valvular se dan principalmente en las aletas mitrales $(65 \%)$, tricuspídeas $(10 \%)$ o en ambas $(25 \%)$. Macroscópicamente se aprecian cambios consistentes con engrosamiento del borde libre, habitualmente en forma de nódulos, opacidad de las valvas y ruptura de las cuerdas tendinosas ${ }^{15}$. Histopatológicamente los cambios se presentan en la matriz extracelular, donde se acumulan mucopolisacáridos y proliferan fibroblastos. Aún no está bien definida la causa por la cual se degenera el aparato valvular aunque se han planteado hipótesis sobre la acción de la serotonina ${ }^{17}$ y metaloproteasas ${ }^{5}$. Causas como migraciones bacterianas de la boca y acciones mecánicas aun no se han establecido mediante procesos de investigación en perros ${ }^{14}$.

La EDV puede causar una insuficiencia cardíaca congestiva en la cual la actividad neurohormonal mediada principalmente por la noradrenalina, angiotensina II y aldosterona generan un remodelamiento cardíaco; como consecuencia de estos cambios fisiopatológicos el animal presenta signos congestivos como edema pulmonar, ascitis y en algunas raras ocasiones edemas periféricos ${ }^{8}$. Los signos de presentación clínica son muy variados y poco precisos con respecto a la enfermedad. El soplo es el primer indicador de la presencia de la enfermedad, aunque en algunos casos los pacientes pueden presentar la enfermedad de forma oculta en ausencia de este signo.

Las investigaciones realizadas sobre el pronóstico de la enfermedad aún no han logrado definir un parámetro puntual sobre su devenir. Cada paciente es afectado por diferentes condiciones particulares y puede responder de manera diferente a los tratamientos médicos instaurados. Algunos trabajos han propuesto factores que pueden modificar el pronóstico; dentro de ellos se encuentran:

A. Elevación de las neurohormonas: se ha demostrado que el aumento de noradrenalina, angiotensina II, aldosterona y péptidos natriuréticos están asociados con el menor tiempo de sobrevida de los individuos con falla cardiaca ${ }^{8}$.

B. Estadio de la falla cardiaca: a medida que avanza la insuficiencia cardíaca las posibilidades de sobrevida disminuyen ${ }^{12}$.

C. Agrandamiento de la aurícula izquierda: por la misma condición de la enfermedad en la cual la regurgitación aumenta la presión auricular y por consiguiente la de los capilares pulmonares, el agrandamiento severo de la aurícula izquierda se asocia con una mayor posibilidad de episodios congestivos refractarios ${ }^{7}$.

D. Medicamentos: la incorporación temprana de medicamentos como espironolactona o pimobendan han mostrado un mayor tiempo de sobrevida ${ }^{6,12}$.

La detección temprana de la enfermedad y por ende la selección adecuada de los tratamientos puede disminuir la morbilidad y mortalidad de la enfermedad valvular. El objetivo de esta investigación fue determinar la presencia de lesiones del aparato valvular mitral y tricuspídeo compatibles con enfermedad degenerativa valvular en perros adultos asintomáticos.

\section{MATERIAL Y MÉTODOS}

Se obtuvo una muestra de 38 corazones de perros eutanasiados en el Centro de Adopción y Tenencia de Mascotas de Bogotá (Colombia) como parte del control poblacional en el programa distrital de control de la rabia. Previo a este procedimiento los animales fueron examinados buscando signos típicos de enfermedad cardiovascular y solamente se seleccionaron animales que no presentaran soplo, ascitis, edema pulmonar o enfermedad sistémica evidente en la valoración.

Inmediatamente a posteriori de la eutanasia se realizó una incisión lateral por el espacio intercostal para extraer el corazón, el cual se almacenó en refrigerador envuelto en bolsas plásticas. Pasados no más de 5 horas se extrajeron los aparatos valvulares mitral y tricuspídeo para ser fijados en formalina buferada al $10 \%$. En el laboratorio se procedió a realizar cortes independientes de la aleta septal y lateral involucrando la pars esponjosa, la pars fibrosa y la superficie atrial. Las láminas fueron coloreadas con hematoxilina eosina y alcian blue.

A cada una de las laminas se les hizo un reconocimiento histológico del órgano, de la arquitectura y del patrón de distribución de la lesión; se tuvo en cuenta el tipo de fibra predominante, el tipo y estado de célula y la cantidad y tipo de infiltración del material mucopolisacárido. Los hallazgos microscópicos se clasificaron teniendo en cuenta los criterios presentados en la Tabla 1. 
Tabla 1. Clasificación histopatológica de las aletas valvulares.

\begin{tabular}{ll}
\hline lesión & \multicolumn{1}{c}{ características microscópicas } \\
\hline grado I & $\begin{array}{l}\text { Se observa un incremento de los mucopolisacáridos en la espongiosa en el medio distal de la valva, con nódulos } \\
\text { espesos a manera de remolinos específicamente por la deposición de los mucopolisacáridos en la fibrosa. Las fibras } \\
\text { de colágeno se encuentran intactas. }\end{array}$ \\
\hline grado II & $\begin{array}{l}\text { El medio distal de la espongiosa valvular exhibe un aumento de poca sustancia mucoide, con leve degeneración de } \\
\text { fibras de colágeno en la pars fibrosa, sin embargo se pueden observar en el medio distal de la arquitectura valvular } \\
\text { en general interrumpidas. }\end{array}$ \\
\hline grado III & $\begin{array}{l}\text { Se observa un marcado aumento de mucopolisacáridos y severa interrupción o ruptura de fibras de colágeno en la } \\
\text { fibrosa en el medio distal de la válvula. El componente de la fibrosa es completamente reemplazado por mucopoli- } \\
\text { sacáridos y proliferación de fibroblastos en la superficie atrial y ventricular. }\end{array}$ \\
\hline
\end{tabular}

Fuentes: modificado de Jubb ${ }^{13}$ y Kogure ${ }^{15}$.

Tabla 2. Clasificación de los hallazgos encontrados en los aparatos valvulares con las coloraciones de hematoxilina/eosina y alcian blue (\%).

\begin{tabular}{lcccccccc}
\hline & HEMS & HEML & HETS & HETL & ABMS & ABML & ABTS & ABTL \\
\hline NP & 34,2 & 31,6 & 44,7 & 15,8 & 23,7 & 23,7 & 31,6 & 34,2 \\
grado I & 28,9 & 26,3 & 23,7 & 36,8 & 31,6 & 31,6 & 31,6 & 31,6 \\
grado II & 28,9 & 31,6 & 10,5 & 31,6 & 28,9 & 36,8 & 21,1 & 18,4 \\
grado III & 7,90 & 10,5 & 21,1 & 15,8 & 7,90 & 10,5 & 21,1 & 15,8 \\
\hline
\end{tabular}

NP: no presenta lesión, HEMS: tinción H\&E de la aleta septal de la mitral, HEML: tinción H\&E de la aleta lateral de la mitral, HETS: tinción H\&E de la aleta septal de la tricúspide, HETL: tinción H\&E de la aleta lateral de la tricúspide, ABMS: tinción alcian blue de la aleta septal de la mitral, ABML: tinción Alcian blue de la aleta lateral de la mitral, ABTS: tinción alcian blue de la aleta septal de la tricuspide, ABTL: tinción alcian blue de la aleta lateral de la tricúspide.

\section{RESULTADOS}

Los perros de los cuales se recolectaron los aparatos valvulares fueron $57,9 \%$ machos, $42,1 \%$ hembras, principalmente mestizos $(n=21)$, Poodle $(n=15)$, Cocker Spaniel $(n=1)$ y Schnauzer $(n=1)$. El peso fue de $7,34 \pm 2,44 \mathrm{~kg}(3-13 \mathrm{~kg})$ y la edad aproximada por evaluación dentaria fue $7 \pm 3$ años (2-15 años).

$\mathrm{Al}$ análisis macroscópico se encontró que el $86,8 \%$ de las aletas mitrales septales (AMS), el 73,7\% de las aletas mitrales laterales (AML), el $63,2 \%$ de las aletas

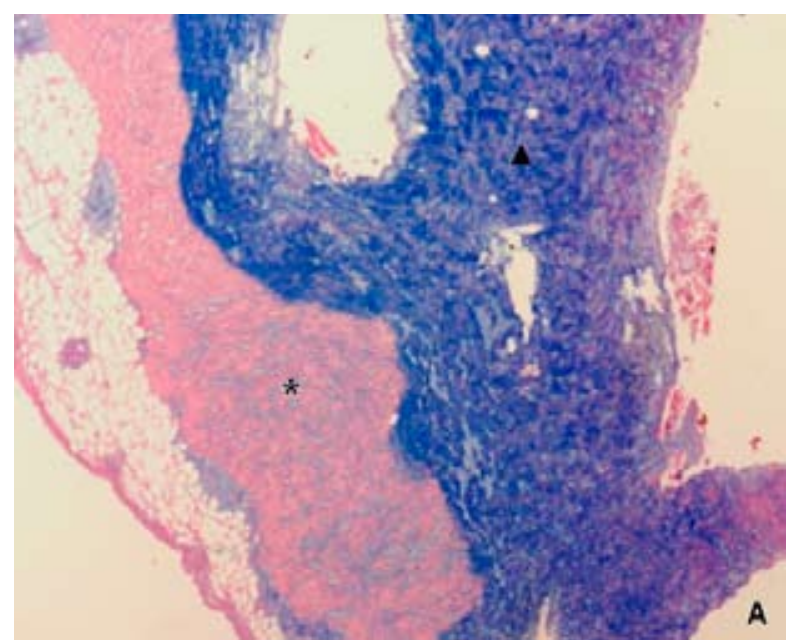

tricuspídeas septales (ATS) y el 65,8\% de las aletas tricuspídeas laterales (ATL) presentaron cambios compatibles con EDV. Con la coloración de hematoxilina y eosina (H\&E) se encontró que un $64,7 \%$ de las AMS y el $67,4 \%$ de las AML presentaron cambios compatibles con EVD al igual que el $55,9 \%$ de las ATS y el $82,4 \%$ de las ATL. Con la coloración alcian blue, especial para evaluar tejido conectivo, se encontró que el $72,7 \%$ de las AMS y el 75,8\% de las AML presentaron cambios compatibles con EVD. De igual manera al evaluar el aparato tricuspídeo se encontró que el $62,5 \%$ de sus

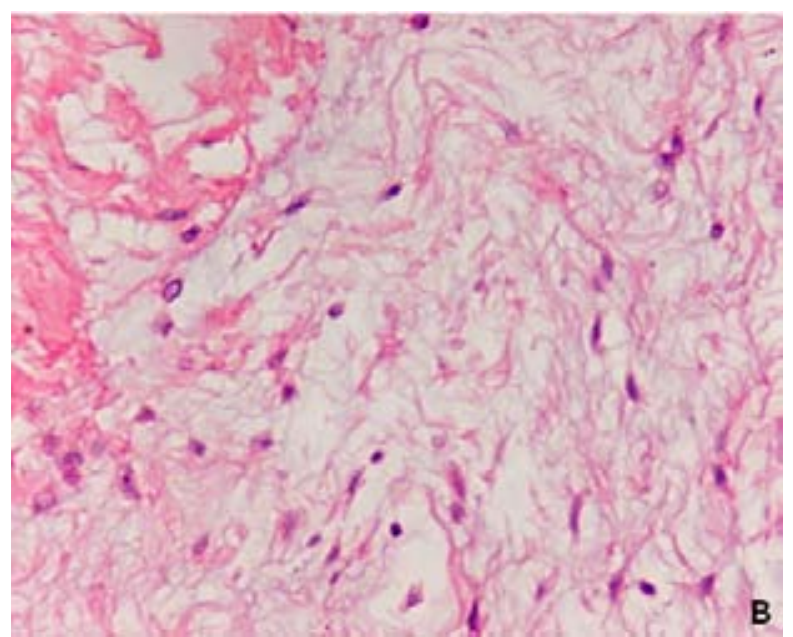

Figura 1. A: tinción alcian blue, valva tricuspídea septal clasificada como grado I. Nótese el aumento de mucopolisacáridos (color azul) en la pars espongiosa (cabeza de flechas), con algunos nódulos espesos (asteriscos) por deposición de mucopolisacáridos en la fibrosa. B: obsérvese que las fibras de colágeno se encuentran intactas. 
aletas septales y el $62,5 \%$ de las aletas laterales presentaron estos cambios (Tabla 2 y Figuras 1, 2 y 3 ).

\section{DISCUSIÓN}

La EDV afecta principalmente a perros con un peso menor de $15 \mathrm{~kg}$ y edades superiores a 7 años ${ }^{2}$. Se sospecha la presencia de esta enfermedad ante la aparición de un soplo mitral o tricuspídeo regurgitativo en perros susceptibles o en animales con signos de congestión como edema pulmonar o ascitis ${ }^{20}$. Las anormalidades morfológicas consisten en un engrosamiento de las aletas valvulares auriculo-ventriculares con posterior agrandamiento de las aurículas y ventrículos según el lado afectado. Para el diagnóstico y clasificación del estadio de la enfermedad se utiliza la radiografía y la ecocardiografía. Esta última es mucho más sensible ya que permite determinar los cambios morfológicos y cuantificar la insuficiencia de los aparatos valvulares mitral y tricuspideo ${ }^{2}$.

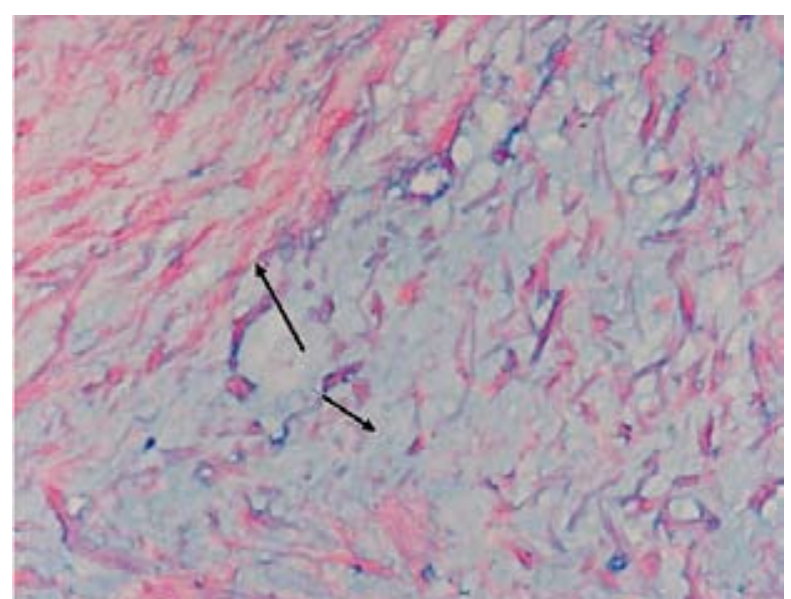

Figura 2. Tinción alcian blue, valva tricuspídea septal clasificada como grado II. Véase el aumento de la sustancia mucoide con leve degeneración de fibras de colágeno en la fibrosa (flechas).

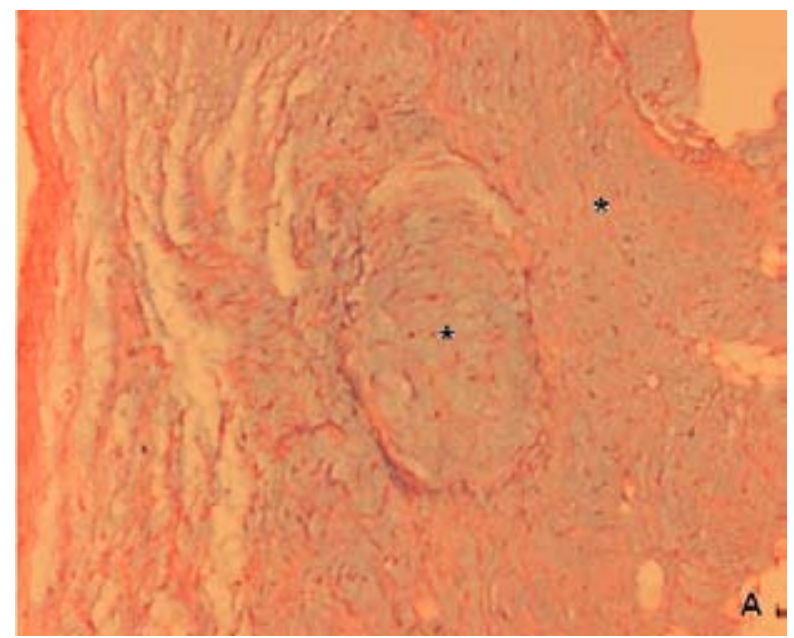

La prevalencia de EDV es alta dentro de las enfermedades cardiovasculares en perros ${ }^{14,20}$. Se genera a partir de pacientes que son diagnosticados cuando presentan signos clínicos, en razas susceptibles como el cavalier King Charles spaniel se ha encontrado que un $52 \%$ de animales entre 4 y 9 años $(n=32)$ presentaron soplos cardíacos indicando lesión mitral o tricuspídea, en ningún caso se ha evaluado a los animales asintomáticos mediante técnicas no invasivas o histopatología ${ }^{10}$.

Se ha planteado que la EDV es heredada siguiendo un modelo poligénico ${ }^{16,19}$. Esto permite inferir que los procesos de deterioro de la matriz extracelular de las aletas valvulares podrían iniciarse antes de la aparición de los síntomas. El cambio en la matriz extracelular como la incorporación de mucopolisacáridos, colágeno y fibroblastos puede estar influenciado por metaloproteinasas, el factor de crecimiento transformador beta y la serotonia ${ }^{4,5,17}$. Aun no se conocen cuales son los factores de riesgo que desencadenan estos procesos moleculares.

En el presente trabajo se encontró que en un grupo de perros asintomáticos la aparición de cambios histopatológicos compatibles con EDV fue del $76,3 \%$ en las aletas valvulares de la mitral y del $67,1 \%$ en las aletas valvulares de la tricúspide, indicando que los cambios estructurales de la matriz extracelular se presentan en ausencia de soplo. Muy poco se ha hablado de una fase oculta de la enfermedad, pero estos hallazgos indican que los cambios microscópicos ocurren en la aletas valvulares muy precozmente y posiblemente mucho antes de la aparición del soplo; por ello se evidencia la necesidad de instaurar programas de prevención para los perros susceptibles, principalmente en razas como Poddle, Cocker, Pinscher, Schnauzer y cavalier, entre otros $^{2}$.

Los factores que desencadenan los procesos moleculares causantes del deterioro valvular son aún desconocidos, pero se sabe que el remodelado ventricular, cambios macroscópicos y microscópicos de los miocitos, es causado por la cadena de neurohormonas que

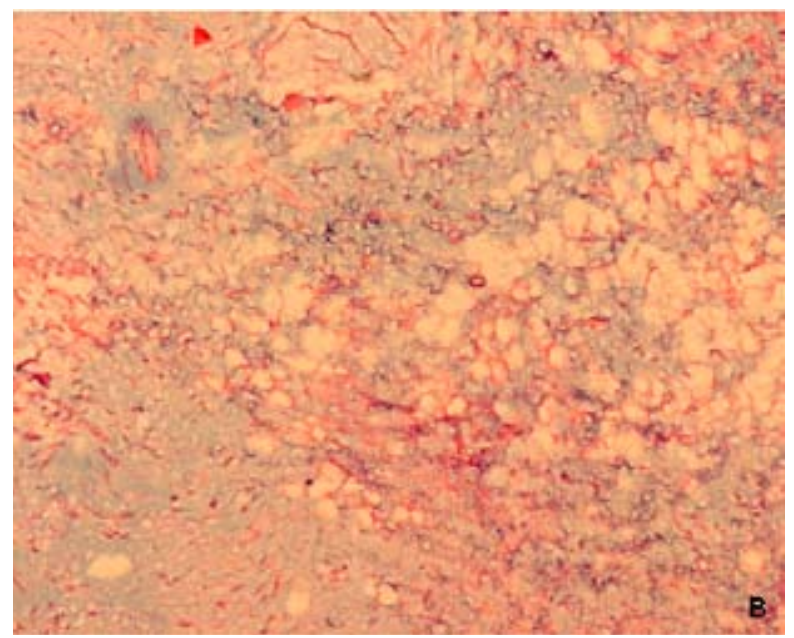

Figura 3. Tinción alcian blue, valva tricuspídea lateral, clasificada como grado III. A: Obsérvese el marcado aumento de mucopolisacáricos e interrupción de las fibras de colágeno en la fibrosa el cual es completamente reemplazado por mucopolisacáricos (asteriscos). B: Nótese la abundante proliferación de fibroblastos. 
se activan como consecuencia de la disminución del gasto cardiaco y la perfusión tisular ${ }^{8}$. Esta descarga hormonal predispone al animal a entrar en una insuficiencia cardiaca congestiva que culmina con la muerte. Trabajos como el RALES o el QUEST han demostrado que el uso de inhibidores de la enzima convertidora de angiotensina y antialdosterónicos amplían la sobrevida de los animales con EDV 2,6,18. Actualmente el uso del pimobendan es una alternativa médica efectiva en la evolución de los animales con EVD ${ }^{3}$. Por lo tanto es posible que la intervención farmacológica en estas etapas tempranas pueda demorar los cambios en las paredes ventriculares y el aparato valvular, disminuyendo la morbilidad y mortalidad.

En este estudio no se observó una predilección por sexo ya que la distribución de los hallazgos fue similar tanto para machos como para hembras. Es posible que la progresión de la enfermedad difiera según el sexo y que exista algún factor desencadenante que sea el responsable de esta diferencia. En los trabajos realizados en cavalier King Charles spaniel, se encontraron cambios en animales jóvenes ( 2 años) lo que hace pesar en modificaciones tempranas de los aparatos valvulares y progresiones lentas de la enfermedad.

La limitación de este estudio consistió en que no se realizaron conjuntamente ecocardiografías que permitieran asociar los cambios encontrados con la presencia de insuficiencias valvulares y la valoración semiológica realizada y de esta manera poder asociar la anormalidad estructural con las condiciones hemodinámicas de cada animal.

Se concluye que los cambios histopatológicos de las válvulas auriculoventriculares compatibles con enfermedad degenerativa valvular están marcadamente presentes en los animales asintomáticos estudiados y que es posible que el proceso fisiopatológico mediado por neurohormonas ocurra mucho antes de la aparición de los síntomas. Por esta razón es importante realizar una valoración preventiva mediante el uso de la ecocardiografia para determinar los cambios en las aletas valvulares y la aparición de insuficiencias valvulares tempranas, así como vigilar o tomar la decisión de instaurar una medicación según sea el caso. Se debe investigar la evolución de la enfermedad en los pacientes con diagnóstico temprano y determinar la existencia de factores de riesgo que aceleren el proceso de aparición de los signos.

Agradecimientos. A la Dirección Nacional de Investigación de la Universidad Antonio Nariño, Bogotá (Colombia), por el financiamiento del estudio.

\section{REFERENCIAS}

1. Atkins CE, Keene BW, Brown WA, Coats JR, Crawford MA, DeFrancesco TC, Edwards NJ, Fox PR, Lehmkuhl LB, Luethy MW, Meurs KM, Petrie JP, Pipers FS, Rosenthal SL, Sidley JA, Straus JH. 2007. Results of the veterinary enalapril trial to prove reduction in onset of heart failure in dogs chronically treated with enalapril alone for compensated, naturally occurring mitral valve insufficiency. $J$ Vet Inter Med 21: 1061-1069.

2. Atkins C, Bonagura J, Ettinger S, Fox P, Gordon S, Haggstrom J, Hamlin R, Keene B, Luis-Fuentes V, Stepien R. 2009. Guidelines for the diagnosis and treatment of canine chronic valvular heart disease. $J$ Vet Inter Med 23: $1142-1150$.

3. Atkinson KJ, Fine DM, Thombs LA, Gorelick JJ, Durham HE. 2009. Evaluation of pimobendan and N-terminal probrain natriuretic peptide in the treatment of pulmonary hypertension secondary to degenerative mitral valve disease in dogs. J Vet Inter Med 23: 1190-1196.

4. Aupperle H, März I, Thielebein J, Schoon HA. 2008. Expression of transforming growth factor-beta 1, beta 2 and beta 3 in normal and diseased canine mitral valves. $J$ Comp Pathol 139: 97-107.

5. Aupperle H, Thielebein J, Kiefer B, März I, Dinges G, Schoon HA, Schubert A. 2009. Expression of genes encoding matrix metalloproteinases (MMPs) and their tissue inhibitors (TIMPs) in normal and diseased canine mitral valves. J Comp Pathol 140: 271-277.

6. Bernay F, Bland JM, Häggström J, Baduel L, Combes B, Lopez A, Kaltsatos V. 2010. Efficacy of spironolactone on survival in dogs with naturally occurring mitral regurgitation caused by myxomatous mitral valve disease. $J$ Vet Intern Med 24: 331-341.

7. Borgarelli M, Savarino P, Crosara S, Santilli RA, Chiavegato $D$, Poggi $M$, Bellino $C$, La Rosa $G$, Zanatta $R$, Haggstrom J, Tarducci A. 2008. Survival characteristics and prognostic variables of dogs with mitral regurgitation attributable to myxomatous valve disease. $J$ Vet Intern Med 22: 120-128.

8. Chatterjee K. 2005. Neurohormonal activation in congestive heart failure and the role of vasopressin. Am J Cardiol 95: 8B-13B.

9. Gómez L. 2004. Degeneración valvular mixomatosa. Analisis estadístico año 2003. Visión Veterinaria (Colombia) 20: 20-24.

10. Häggström J, Hansson K, Kvart C, Swenson L. 1992. Chronic valvular disease in the cavalier King Charles spaniel in Sweden. Vet Rec 131: 549-553.

11. Häggström J, Duelund H, Kvart C. 2004. New insights into degenerative mitral valve disease in dogs. Vet Clin North Am Small Anim Pract 34: 1209-1226.

12. Häggström J, Boswood A, O'Grady M, Jöns O, Smith S, Swift S, Borgarelli M, Gavaghan B (et al.). 2008. Effect of pimobendan or benazepril hydrochloride on survival times in dogs with congestive heart failure caused by naturally occurring myxomatous mitral valve disease: the Quest study. J Vet Inter Med 22: 1124-1135.

13. Jubb KV, Kennedy PC, Palmer N. 2007. Pathology of domestic animals, 5th ed, vol. 3, Saunders, Philadelphia, 2340 p.

14. Kittleson M. 1999. Degeneración mixomatosa de las válvulas auriculo-ventriculares. En: Medicina cardiovascular de pequeños animales (Kittleson M. Ed), Multimédica, Barcelona, p. 297-318. 
15. Kogure K. 1980. Pathology of chronic mitral valvular disease in the dog. Nippon Juigaku Zasshi 42: 323-335.

16. Olsen LH, Fredholm M, Pedersen HD. 1999. Epidemiology and inheritance of mitral valve prolapse in Dachshunds. $J$ Vet Inter Med 13: 448-456.

17. Oyama MA, Levy RJ. 2010. Insights into serotonin signaling mechanisms associated with canine degenerative mitral valve disease. J Vet Inter Med 24: 2-8.
18. Shafiq MM, Miller AB. 2009. Blocking aldosterone in heart failure. Ther Adv Cardiovasc Dis 3: 379-385.

19. Swift S. 1996. The problem of inherited diseases. 5: Valvular disease in Cavalier King Charles spaniels. $J$ Small Anim Pract 37: 505-506.

20. Ware W. 2007. Acquired valve diseases. In: Cardiovascular disease in small animal medicine (Ware W Ed.), Mason, London, p. 263-279.

\section{Revista Veterinaria obtuvo el máximo nivel de categorización del CAICYT-CONICET}

Tras el pertinente proceso de evaluación según criterios de calidad editorial, en setiembre de 2005 CAICYT-CONICET ha clasificado a nuestra publicación con Categoría 1 (nivel superior de excelencia), con lo cual pasa a integrar el Catálogo Latindex (folio 14022). La Dirección de Revista veterinaria agradece a quienes colaboraron para obtener tan importante distinción. Ver: http://www.latindex. unam.mx/busquedas/catalogotitulo.html 